Tạp chí Khoa học và Công nghệ biển T11 (2011). Số 1. Tr 31 - 42

\title{
TÍNH TƯơng TÁC GIŨ๋A Hệ ỐNG DÃN ĐÔI VÀ NỀN SAN HÔ DƯớI TÁC DỤNG CỦA TẢI TRỌNG NỔ
}

\author{
LÊ TÂN
}

\section{Học viện Kỹ thuật Quân sự}

\begin{abstract}
Tóm tắt: Bài báo trình bày phương pháp tính tương tác giữa hẹ 2 ống dẫn và nền san hô chịu tác dụng của tải trọng nổ do bom đạn gây nên. Bài toán được giải quyết theo mô hình biến dạng phẳng, với việc ứng dụng phưong pháp phần tử hưu hạn (PTHH), kết hợp phần biến dạng phẳng và phà̀n tử tiếp xúc hai chiều (2D). Kết hợp tích phân trực tiếp Newmark và phuơng pháp lặp Newton-Raphson tác giả đã xây dựng thuật toán và lập trình trong môi truờng Matlab để giải phwơng trình chuyển động dạng phi tuyến liên kết của hệ. Nội dung của bài báo có thể làm tài liệu tham khảo cho việc nghiên cúu và tính toán, lựa chọn phuơng án tối uu cho hệ ống dẫn làm việc trong nền san hô.
\end{abstract}

\section{MỞ ĐÀ̀U}

Khi bom đạn nổ thì quá trình thực hiện phản ứng lan truyền cực nhanh trong thời gian ngắn kèm theo là một lượng lớn năng lượng được giải phóng từ trong một thể tích hạn chế của khối nổ lan toả ra xung quanh tác động vào mọi vật. Sự giải phóng năng lượng đột ngột làm cho nhiệt độ và áp suất tăng, đồng thời sản phẩm nổ dãn nở làm cho không khí bị nén mạnh tạo thành sóng xung kích (SXK). Sóng xung kích gồm 2 vùng: vùng pha nén và vùng pha dãn (hình 1 ).

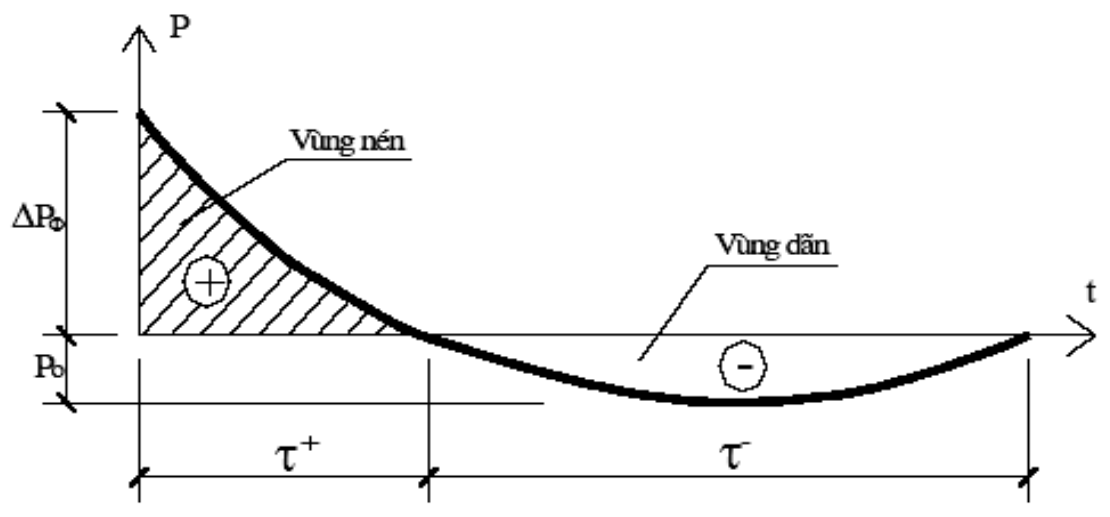

Hình 1: Biểu đồ sóng xung kích 
Vì áp suất pha nén lớn gấp nhiều lần áp suất pha dãn nên trong thực tế thường dùng pha nén, với các tham số $\Delta \mathrm{P}_{\Phi}$ và $\tau^{+}$để tính toán công trình.

Khi chịu tác dụng của SXK do những vụ nổ mạnh, như thuốc nổ cường độ lớn, bom hạt nhân,... thì thời gian kết cấu công trình chịu tải trọng cực đại nhỏ hơn $\tau^{+}$, do vậy có thể coi áp suất thay đổi theo đường thẳng tiếp tuyến với đường cong $\Delta \mathrm{P}_{\Phi}$ tại thời điểm $\mathrm{t}=0$ và quy luật tải trọng lúc đó được mô tả theo biểu thức [4]:

$$
\Delta \mathrm{p}(\mathrm{t})=\Delta \mathrm{p}_{\Phi}\left(1-\frac{\mathrm{t}}{\tau}\right)
$$

Việc nghiên cứu, tính toán kết cấu công trình chịu tác dụng của dạng tải trọng này là vấn đề phức tạp và rất cần thiết. Vì vậy, nghiên cứu tính toán hệ ống dẫn làm việc trong nền san hô chịu tác dụng của sóng xung kích do bom đạn nổ gây ra là điều rất có ý nghĩa.

\section{CÁC GIẢ THIẾT, MÔ HÌNH VÀ PHƯƠNG PHÁP TÍNH}

\section{Các giả thiết và mô hình tính của bài toán}

Bài toán được giải quyết dựa vào các giả thiết sau:

- Hệ ống dẫn là đàn hồi, biến dạng tuyến tính.

- Mỗi lớp nền san hô là vật liệu đồng nhất, đẳng hướng, đàn hồi tuyến tính. Quá trình hệ ống dẫn làm việc, không có hiện tượng tách, trượt giữa các lớp nền với nhau.

- Hệ ống dẫn và nền san hô làm việc trong điều kiện biến dạng phẳng. Liên kết giữa hệ ống dẫn và nền san hô được thay thế bằng liên kết nút giữa các phần tử biến dạng phẳng $2 \mathrm{D}$ thông qua liên kết với phần tử tiếp xúc Goodman [3]. Liên kết tiếp xúc giữa hệ ống dẫn và nền san hô là liên kết một chiều.

- Khi tính toán, đối với vật liệu san hô, bỏ qua lực dính giữa kết cấu ống dẫn và nền.

Tách từ hệ thực bán vô hạn ra một miền hữu hạn bao gồm hệ ống dẫn và một phần nền gọi là miền nghiên cứu, trên biên miền nghiên cứu được đặt các liên kết, việc tính toán được thực hiện trên miền nghiên cứu đã xác định. Kích thước biên của miền nghiên cứu được xác định theo phương pháp lặp $[1,2]$ (hình 2).

\section{Phương pháp tính và các loại PTHH}

Để tính toán kết cấu theo mô hình nói trên, tác giả sử dụng phương pháp PTHH, trong đó các loại phần tử sử dụng là: Đối với nền san hô và hệ ống dẫn sử dụng kết hợp loại phần tử tứ giác đẳng tham số 4 nút và phần tử tam giác phẳng 3 điểm nút. Đối với lớp 
tiếp xúc giữa hệ ống dẫn và nền san hô sử dụng loại phần tử tiếp xúc tứ giác 4 điểm nút (four nodes slip element).

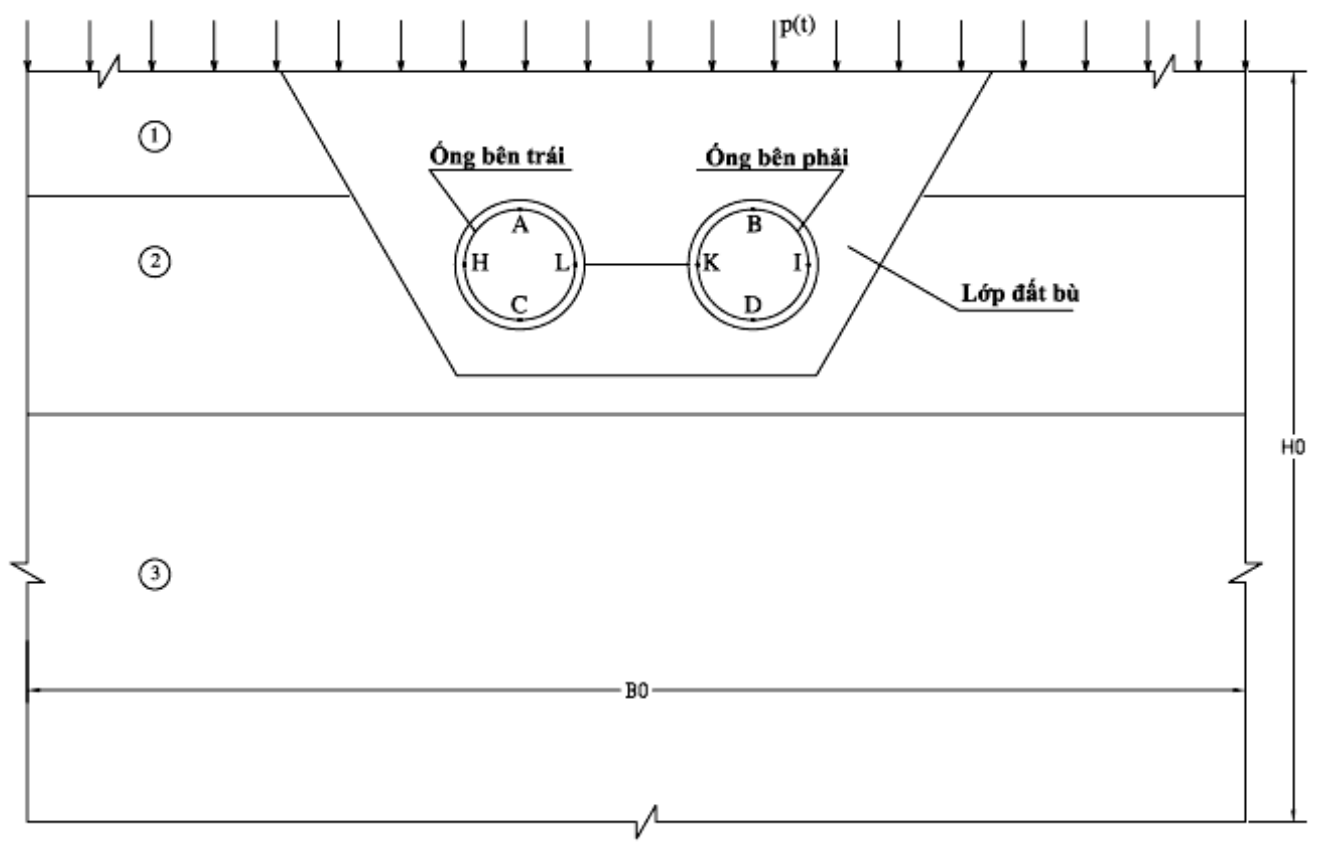

Hình 2: Mô hình thực của bài toán

\section{THIẾT LẬP CÁC PHƯƠNG TRÌNH CƠ BẢN BÀ̀NG PHƯƠNG PHÁP PTHH}

\section{Các quan hệ đối với phần tử thuộc nền và kết cấu}

Đối với phần tử phẳng dạng tứ giác 4 điểm nút và dạng phần tử phẳng tam giác 3 điểm nút, chuyển vị tại một điểm bất kỳ trong phạm vi phần tử được nội suy từ véc tơ chuyển vị nút của phần tử theo biểu thức [2]:

$$
\{\mathrm{u}\}=[\mathrm{N}]_{\mathrm{m}}\{\mathrm{U}\}_{\mathrm{m}}
$$

Trong đó:

$\{u\}=\left\{\begin{array}{ll}u_{x} & u_{y}\end{array}\right\}^{T}-$ véc tơ chuyển vị tại điểm $(x, y)$ thuộc phần tử,

$[\mathrm{N}]_{\mathrm{m}}$ - ma trận hàm dạng của phần tử thứ $m$,

$\{\mathrm{U}\}_{\mathrm{m}}$ - véc tơ chuyển vị nút của phần tử thứ $m$.

Véc tơ biến dạng tại một điểm của phần tử $\{\varepsilon\}$ được viết như sau: 
$\{\varepsilon\}=[\mathrm{B}]_{\mathrm{m}}\{\mathrm{U}\}_{\mathrm{m}}$

Trong đó $[\mathrm{B}]_{\mathrm{m}}$ là ma trận biến dạng - chuyển vị của phần tử.

Ma trận độ cứng phần tử được xác định theo biểu thức sau:

$[\mathrm{K}]_{\mathrm{m}}=\int_{\mathrm{A}_{\mathrm{m}}} \mathrm{h}[\mathrm{B}]_{\mathrm{m}}^{\mathrm{T}}[\mathrm{D}][\mathrm{B}]_{\mathrm{m}} \mathrm{dA} \mathrm{A}_{\mathrm{m}}$

với [D] là ma trận vật liệu phần tử, phụ thuộc vào modul Young E, hệ số Possion v.

Sử dụng phương phương pháp tích phân số cầu phương Gauss-Legendre, biểu thứ (4) được viết lại: $[\mathrm{K}]_{\mathrm{m}}=\mathrm{h} \sum_{\mathrm{i}=1}^{\mathrm{n}}[\mathrm{B}]_{\mathrm{m}, \mathrm{i}}^{\mathrm{T}}[\mathrm{D}][\mathrm{B}]_{\mathrm{m}, \mathrm{i}} \operatorname{det} \mathrm{J}_{\mathrm{i}} \mid \mathrm{W}_{\mathrm{li}} \mathrm{W}_{2 \mathrm{i}}$

Trong đó: $i$ - điểm tích phân thứ $i$ có toạ độ $\left(r_{\mathrm{i}}, s_{\mathrm{i}}\right), n$ - tổng số điểm tích phân, $\operatorname{det}|\mathrm{J}|$ - định thức của ma trận Jacobi, $W_{1 i}, W_{2 i}$ - trọng số của phép tích phân cầu phương, chỉ số $i$ biểu diễn giá trị của hàm tại điểm Gauss thứ $i$.

Ma trận khối lượng của phần tử được xác định theo công thức sau:

$$
[\mathrm{M}]_{\mathrm{m}}=\int_{\mathrm{A}_{\mathrm{m}}} \rho \mathrm{h}[\mathrm{N}]_{\mathrm{m}}^{\mathrm{T}}[\mathrm{N}]_{\mathrm{m}} \mathrm{dA} \mathrm{m}_{\mathrm{m}}
$$

và cũng được tính theo phương pháp tích phân số cầu phương Gauss-Legendre.

Trong đó: $\rho$ - khối lượng riêng vật liệu của phần tử thứ $m ; A_{\mathrm{m}}$ - diện tích phần tử thứ $m ; h$ - chiều dày phần tử,

\section{Các quan hệ đối với phần tử thuộc lớp tiếp xúc (PTTX)}

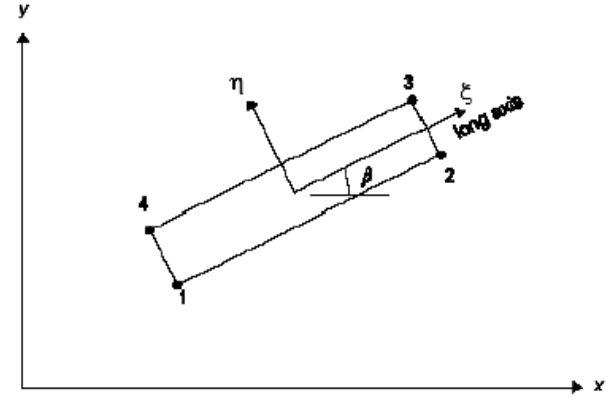

(a)

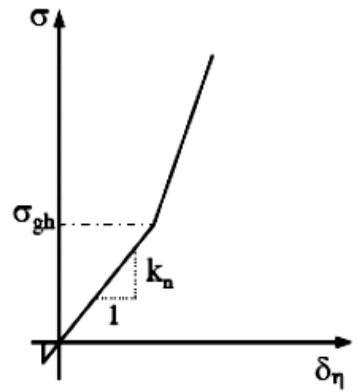

(b)

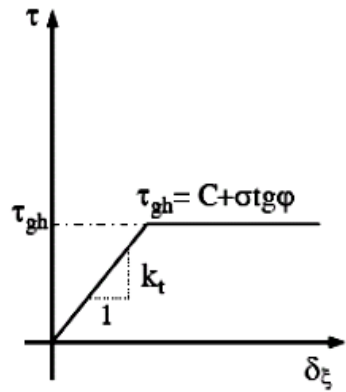

(c)

Hình 3: Mô hình phần tử tiếp xúc Goodman. a) Sơ đồ hình học của phần tử tiếp xúc; b) Quan hệ ứng suất pháp tuyến với biến dạng pháp tuyến; c) Quan hệ ứng suất tiếp tuyến với biến dạng tiếp tuyến 
Phần tử tiếp xúc 2 chiều được bố trí vào lớp tiếp xúc giữa ống dẫn và nền san hô thể hiện tính chất liên kết một chiều của nền. Mô hình phần tử tiếp xúc có chiều dày "bằng không" được chỉ ra như trên hình 3 . Do chiều dày $\mathrm{t}$ của phần tử gần như bằng 0 nên các cặp nút 1 và 4,2 và 3,5 và 6 có cùng toạ độ.

Quan hệ giữa số gia ứng suất và số gia biến dạng: $\left\{\begin{array}{c}\Delta \sigma \\ \Delta \tau\end{array}\right\}=\left[\mathrm{D}_{\mathrm{se}}\right\}\left\{\begin{array}{c}\Delta \varepsilon \\ \Delta \gamma\end{array}\right\}$

Trong đó ma trận $\left[\mathrm{D}_{\mathrm{se}}\right]$ được xác định theo biểu thức: $\left[\mathrm{D}_{\mathrm{se}}\right]=\left[\begin{array}{cc}\mathrm{k}_{\eta} & 0 \\ 0 & \mathrm{k}_{\xi}\end{array}\right]$

với: $\mathrm{k}_{\eta}$ và $\mathrm{k}_{\xi}$ tương ứng là độ cứng pháp tuyến và độ cứng tiếp tuyến của phần tử. Các hệ số này có thể được xác định bằng thực nghiệm hoặc xác định thông qua các đặc trưng của nền $[1,3]: \mathrm{k}_{\eta}=\frac{E(1-v)}{(1+v)(1-2 v)} ; \mathrm{k}_{\xi}=\mathrm{G}=\frac{\mathrm{E}}{2(1+v)}$

với: $v$ và $E$ tương ứng là hệ số Poisson và mô đun Young của vật liệu nền.

Chuyển vị một điểm bất kỳ của phần tử trong hệ trục toạ độ địa phương được xác định thông qua chuyển vị của nó trong hệ toạ độ tổng thể và góc lệch trục $\alpha$ :

$\left\{\begin{array}{l}\mathrm{u}_{1} \\ \mathrm{v}_{1}\end{array}\right\}=\left[\begin{array}{cc}\cos \beta & \sin \beta \\ -\sin \beta & \cos \beta\end{array}\right]\left\{\begin{array}{l}\mathrm{u} \\ \mathrm{v}\end{array}\right\}$

Ma trận độ cứng trong hệ toạ độ chung: $\left[\mathrm{K}_{\mathrm{se}}\right]=\iint\left[\mathrm{B}_{\mathrm{se}}\right]^{\mathrm{T}}\left[\mathrm{D}_{\mathrm{se}}\right]\left[\mathrm{B}_{\mathrm{se}}\right] \mathrm{dxdy}$

Trong đó: $\left[\mathrm{B}_{\mathrm{se}}\right]=\left[\begin{array}{cc}\cos \beta & -\sin \beta \\ \sin \beta & \cos \beta\end{array}\right]\left[\mathrm{B}_{\text {local }}\right]$

Đối với PTTX 4 điểm nút, biến dạng trong phần tử là đều, ta có:

$\left[\mathrm{B}_{\text {local }}\right]=\left[\begin{array}{cccccccc}0 & \mathrm{~N}_{1}^{\prime} & 0 & \mathrm{~N}_{2}^{\prime} & 0 & -\mathrm{N}_{3}^{\prime} & 0 & -\mathrm{N}_{4}^{\prime} \\ \mathrm{N}_{1}^{\prime} & 0 & \mathrm{~N}_{2}^{\prime} & 0 & -\mathrm{N}_{3}^{\prime} & 0 & -\mathrm{N}_{4}^{\prime} & 0\end{array}\right]$

với: $\mathrm{N}_{\mathrm{i}}^{\prime}=\frac{\mathrm{dN}_{\mathrm{i}}}{\mathrm{d} \xi}, \xi(T u ̛ ̀ ~-1 ~$ đến +1$)$ dọc theo chiều dài phần tử, $\mathrm{N}_{\mathrm{i}}$ là hàm dạng của PTTX $[1,3]$.

Sử dụng phép biến đổi $\iint \mathrm{dxdy}=\int_{-1}^{1} \operatorname{det}|J| \mathrm{d} \xi$, ma trận độ cứng PTTX được xác định: 


$$
\left[\mathrm{K}_{\mathrm{se}}\right]=\int_{-1}^{1}\left[\mathrm{~B}_{\mathrm{se}}\right]^{\mathrm{T}}\left[\mathrm{D}_{\mathrm{se}}\right]\left[\mathrm{B}_{\mathrm{se}}\right] \operatorname{det}|\mathrm{J}| \mathrm{d} \xi
$$

Trong đó [J] là ma trận Jacobin [1,3].

Ma trận độ cứng của PTTX trong công thức (13) được xác định bằng phương pháp tích phân số cầu phương Gauss-Legendre và sử dụng sơ đồ 1 điểm tích phân $(\xi=0, w=2)$.

\section{Phương trình chuyển động của kết cấu}

Dưới tác dụng của tải trọng, phương trình chuyển động của hệ được viết dưới dạng [2]:

$$
[\overline{\mathrm{M}}][\ddot{\overline{\mathrm{U}}}\}_{+}[\overline{\mathrm{C}}][\dot{\overline{\mathrm{U}}}\}_{+}[\overline{\mathrm{K}}][\overline{\mathrm{U}}\}=\{\overline{\mathrm{R}}\} \text {, }
$$

Trong đó: $[\overline{\mathrm{M}}],[\overline{\mathrm{C}}]$ và $[\overline{\mathrm{K}}]$ tương ứng là ma trận khối lượng, ma trận cản và ma trận độ cứng của hệ, $\{\overline{\mathrm{R}}\}$ là véc tơ tải trọng nút do tải trọng đã cho gây ra.

\section{THUẬT TOÁN GIẢI PHƯƠNG TRÌNH CHUYỄN ĐộNG CỦA KẾT CÂUU}

Sau khi đưa vào các điều kiện biên, (14) có dạng như sau [2]:

$$
[\mathrm{M}]\{\ddot{\mathrm{U}}\}+[\mathrm{C}]\{\dot{\mathrm{U}}\}+[\mathrm{K}]\{\mathrm{U}\}=\{\mathrm{R}\},
$$

Trong thuật toán, để kể đến tính chất liên kết một chiều của nền san hô, các giá trị độ cứng pháp tuyến $\mathrm{k}_{\eta}$ và độ cứng tiếp tuyến $\mathrm{k}_{\xi}$ phụ thuộc vào chuyển vị của các nút chung giữa PTTX và ống dẫn $[1,3]$, nên ma trận độ cứng PTTX $\left[K_{\mathrm{se}}\right]$ phụ thuộc vào véc tơ chuyển vị nút $\{\mathrm{U}\}$, do đó ma trận độ cứng tổng thể $[\mathrm{K}]$ cũng phụ thuộc vào $\{\mathrm{U}\}$. Vì vậy phương trình (15) là phương trình phi tuyến hình học và được viết lại dưới dạng:

$$
[\mathrm{M}][\ddot{\mathrm{U}}\}+[\mathrm{C}(\{\mathrm{U}\})]\{\dot{\mathrm{U}}\}+[\mathrm{K}(\{\mathrm{U}\})]\{\mathrm{U}\}=\{\mathrm{R}\}
$$

Ma trận cản tổng thể được xác định:

$$
[\mathrm{C}]=\alpha[\mathrm{M}]+\beta[\mathrm{K}]=[\mathrm{C}(\{\mathrm{U}\})], \text { với: } \beta=\frac{2 \xi}{\omega_{1}+\omega_{2}} ; \alpha=\beta \omega_{1} \omega_{2} ;\left(\omega_{1}, \omega_{2} \text { là } 2\right. \text { tần số đầu tiên }
$$
trong dao động tự do, $\xi$ là tỷ số cản).

Để giải phương trình chuyển động (16) tác giả kết hợp phương pháp tích phân trực tiếp Newmark với phương pháp lặp Newton - Raphson. Sử dụng phương pháp ma trận độ cứng hiệu quả và véc tơ tải trọng hiệu quả, véc tơ chuyển vị nút tại thời điểm $\mathrm{t}+\Delta \mathrm{t}$ được xác định thông qua số gia chuyển vị nút như sau:

$$
\left[\mathrm{K}_{\mathrm{t}+\Delta \mathrm{t}}^{*}\right][\Delta \mathrm{U}\}^{(\mathrm{i})}=\left\{\mathrm{R}_{\mathrm{t}+\Delta \mathrm{t}}^{*}\right\}^{(\mathrm{i}-1)}-\left\{\mathrm{F}_{\mathrm{t}+\Delta \mathrm{t}}\right\}^{(\mathrm{i}-1)},
$$




$$
\left\{\mathrm{U}_{\mathrm{t}+\Delta t}\right\}^{(\mathrm{i})}=\left\{\mathrm{U}_{\mathrm{t}+\Delta \mathrm{t}}\right\}^{(\mathrm{i}-1)}+\{\Delta \mathrm{U}\}^{(\mathrm{i})},
$$

Việc tính lặp được thực hiện với điều kiện hội tụ theo chuyển vị nút:

$$
\frac{\left\|\{\Delta \mathrm{U}\}^{(\mathrm{i})}\right\|}{\left\|\left\{\mathrm{U}_{\mathrm{t}+\Delta \mathrm{t}}\right\}^{(\mathrm{i})}-\left\{\mathrm{U}_{\mathrm{t}}\right\}\right\|} \leq \varepsilon_{\mathrm{D}}
$$

Véc tơ gia tốc và vận tốc nút tại thời điểm $\mathrm{t}+\Delta \mathrm{t}$ được xác định:

$$
\begin{aligned}
& \left\{\ddot{U}_{t+\Delta t}\right\}=a_{0}\left(\left\{\mathrm{U}_{\mathrm{t}+\Delta t}\right\}-\left\{\mathrm{U}_{\mathrm{t}}\right\}\right)-\mathrm{a}_{2}\left\{\dot{\mathrm{U}}_{\mathrm{t}}\right\}-\mathrm{a}_{3}\left\{\ddot{\mathrm{U}}_{\mathrm{t}}\right\} \\
& \left\{\dot{\mathrm{U}}_{\mathrm{t}+\Delta t}\right\}=\left\{\dot{\mathrm{U}}_{\mathrm{t}}\right\}+\mathrm{a}_{6}\left\{\ddot{\mathrm{U}}_{\mathrm{t}}\right\}+\mathrm{a}_{7}\left\{\ddot{\mathrm{U}}_{\mathrm{t}+\Delta t}\right\}
\end{aligned}
$$

Tóm tắt các bước của thuật giải thể hiện như trong bảng 1 .

Bảng 1: Tóm tắt thuật toán giải bài toán

\section{Nhập dữ liệu ban đầu:}

1. Sơ đồ, kích thước kết cấu

2. Đặc trưng vật liệu

3. Nhập tải trọng

\section{Tính toán so bộ ban đầu:}

1. Tính các ma trận cơ bản: [K], [M], [C].

2. Xác định bước tích phân $\Delta \mathrm{t}$ và các tham số tích phân $\alpha, \delta$. Tính các hệ số của phương pháp tích phân: $a_{0}, a_{1}, a_{2}, a_{3}, a_{4}, \ldots, a_{7}$.

3. Xác định các véc tơ điều kiện ban đầu: $\left\{\mathrm{U}_{0}\right\},\left\{\dot{\mathrm{U}}_{0}\right\},\left\{\ddot{\mathrm{U}}_{0}\right\}$.

4. Tính ma trận độ cứng hiệu quả $\left[\mathrm{K}^{*}\right]$.

II. Tính lặp cho các bước tích phân tại các thời điểm: $t=\Delta t, t=2 \Delta t, t=3 \Delta t, \ldots$ theo các bước sau:

1. Tính véc tơ tải trọng hiệu quả $\left\{\mathrm{R}_{\mathrm{t}+\Delta \mathrm{t}}^{*}\right\}$ tại thời điểm $\mathrm{t}+\Delta \mathrm{t}$

2. Dùng phương pháp lặp xác định véc tơ chuyển vị nút $\left\{\mathrm{U}_{\mathrm{t}+\Delta t}\right\}$ tại thời điểm $\mathrm{t}+\Delta \mathrm{t}$ theo phương trình $(17,18)$. Kiểm tra điều kiện hội tụ $(19)$.

3. Xác định các véc tơ gia tốc và vận tốc tại thời điểm $\mathrm{t}+\Delta \mathrm{t}$ theo (20) 
4. Tính ứng suất, biến dạng và nội lực tại thời điểm $\mathrm{t}+\Delta \mathrm{t}$.

5. Kiểm tra điều kiện tách, trượt, cập nhật ma trận phần tử.

5. Tăng bước thời gian $\mathrm{t}=\mathrm{t}+\Delta \mathrm{t}$ và tiến hành lặp lại từ bước 1 .

\section{VÍ DỤ SỐ}

Trên cơ sở thuật toán đã thiết lập, tác giả lập chương trình tính có tên là Pipes_2010. Chương trình được viết bằng ngôn ngữ MATLAB. Tính toán với hệ ống dẫn đôi trong nền san hô chịu tác dụng của tải trọng sóng xung kích phân bố đều trên bề mặt nền (hình 4).

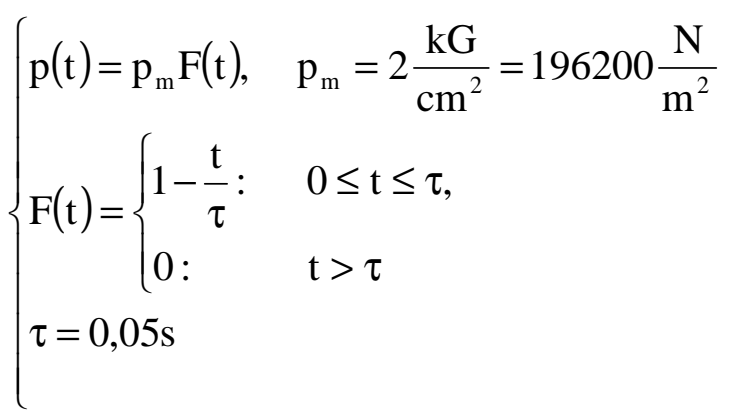

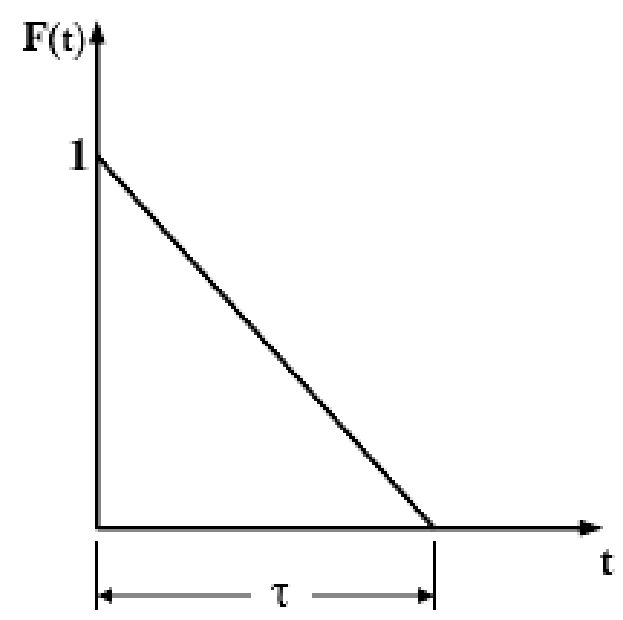

Hình 4: Hàm thời gian của tải trọng

- Kích thước của kết cấu: Hệ 2 ống dẫn giống nhau, đường kính ngoài $\mathrm{D}=1,2 \mathrm{~m}$, đường kính trong $\mathrm{d}=0,8 \mathrm{~m}$ đặt trong nền san hô ở độ sâu so với mặt nền $\mathrm{h}_{0}=4 \mathrm{~m}$. Vật liệu ống làm bằng thép, có $\mathrm{E}=2,1.10^{7} \mathrm{~N} / \mathrm{cm}^{2}, v=0,3 \mathrm{v} \mu$ khèi 1-îng ria ${ }^{\mathrm{a}} \mathrm{g} \rho=7,8 \cdot 10^{-3} \mathrm{~kg} / \mathrm{cm}^{3}$. 
- Sau khi lặp, xác định được kích thước miền tính toán: $\mathrm{B}_{0}=25 \mathrm{~m}$, chiều cao $\mathrm{H}_{0}=$ $15 \mathrm{~m}$, với sai số chuyển vị $\varepsilon=1,74 \%$, tương ứng với 3 lớp nền (thứ tự từ 1 đến 3 ) có đặc trưng cơ lý như bảng 2 (số liệu thí nghiệm tại đảo Song Tử Tây) [1].

Bảng 2: Thuộc tính vật liệu nền san hô [1]

\begin{tabular}{|c|c|c|c|c|c|c|}
\hline Lớp & $\begin{array}{c}\text { Độ sâu } \\
\text { (m) }\end{array}$ & $\begin{array}{c}E \\
\left(N / \mathrm{cm}^{2}\right)\end{array}$ & v & $\begin{array}{c}\rho \\
\left(\mathrm{kg} / \mathrm{cm}^{3}\right)\end{array}$ & $\begin{array}{c}\text { Hệ số ma sát } \\
\text { f }\end{array}$ & Hệ số giảm chấn $\xi$ \\
\hline 1 & 3 & $1,83.10^{4}$ & 0,46 & $2,2.10^{-3}$ & 0,21 & \multirow{3}{*}{0,05} \\
\hline 2 & 7 & $5,19.10^{4}$ & 0,38 & $2,6.10^{-3}$ & 0,32 & \\
\hline 3 & 15 & $8,63 \cdot 10^{4}$ & 0,27 & $2,7 \cdot 10^{-3}$ & 0,41 & \\
\hline
\end{tabular}

Mô hình bài toán thể hiện như trên hình 2.

\section{Kết quả tính:}

Hình 5 và 6 là các kết quả đáp ứng chuyển vị theo thời gian của các điểm tính thuộc hệ ống dẫn.

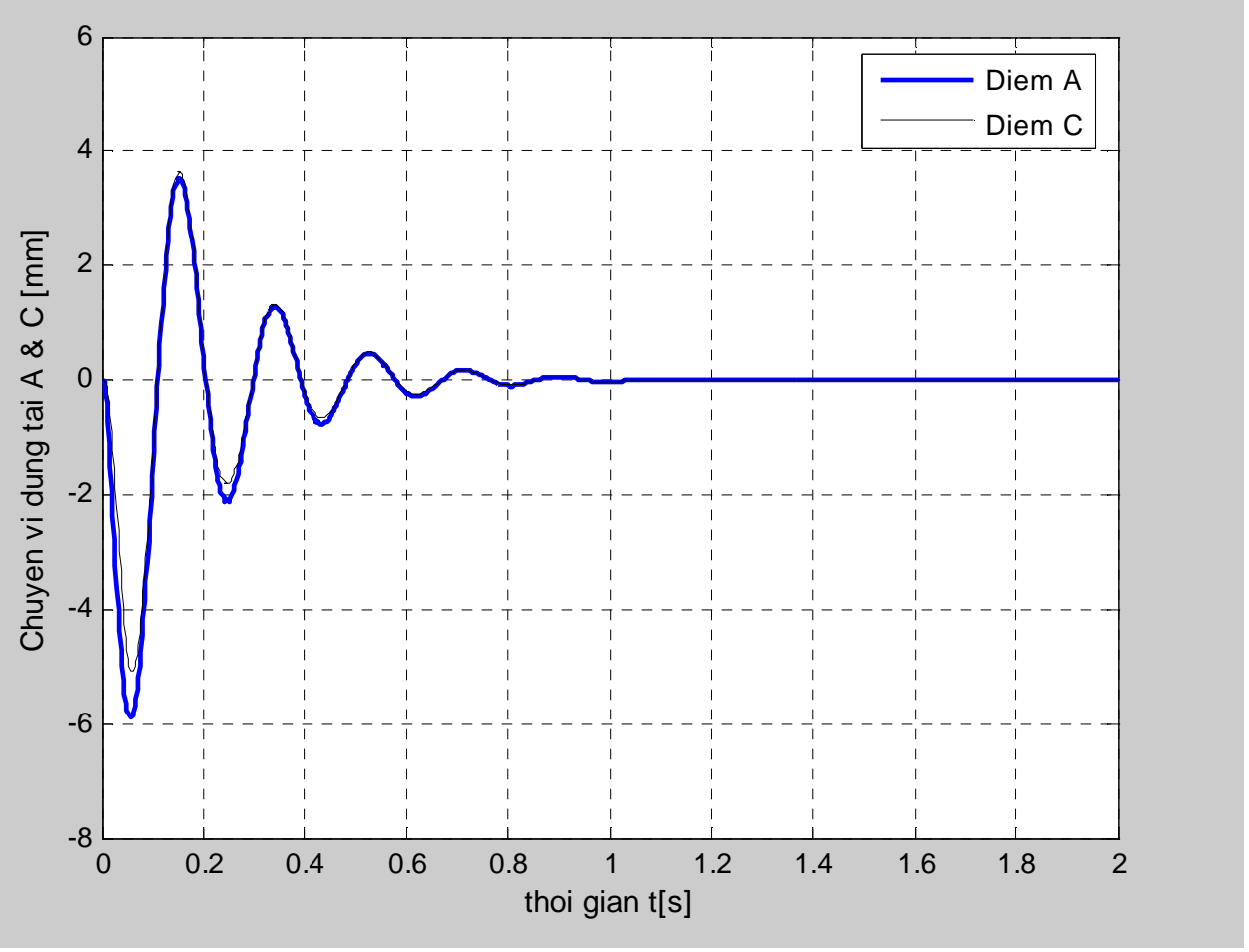

Hình 5: Biến thiên chuyển vị đứng tại điểm $\mathrm{A}$ và $\mathrm{C}$ thuộc ống bên trái 


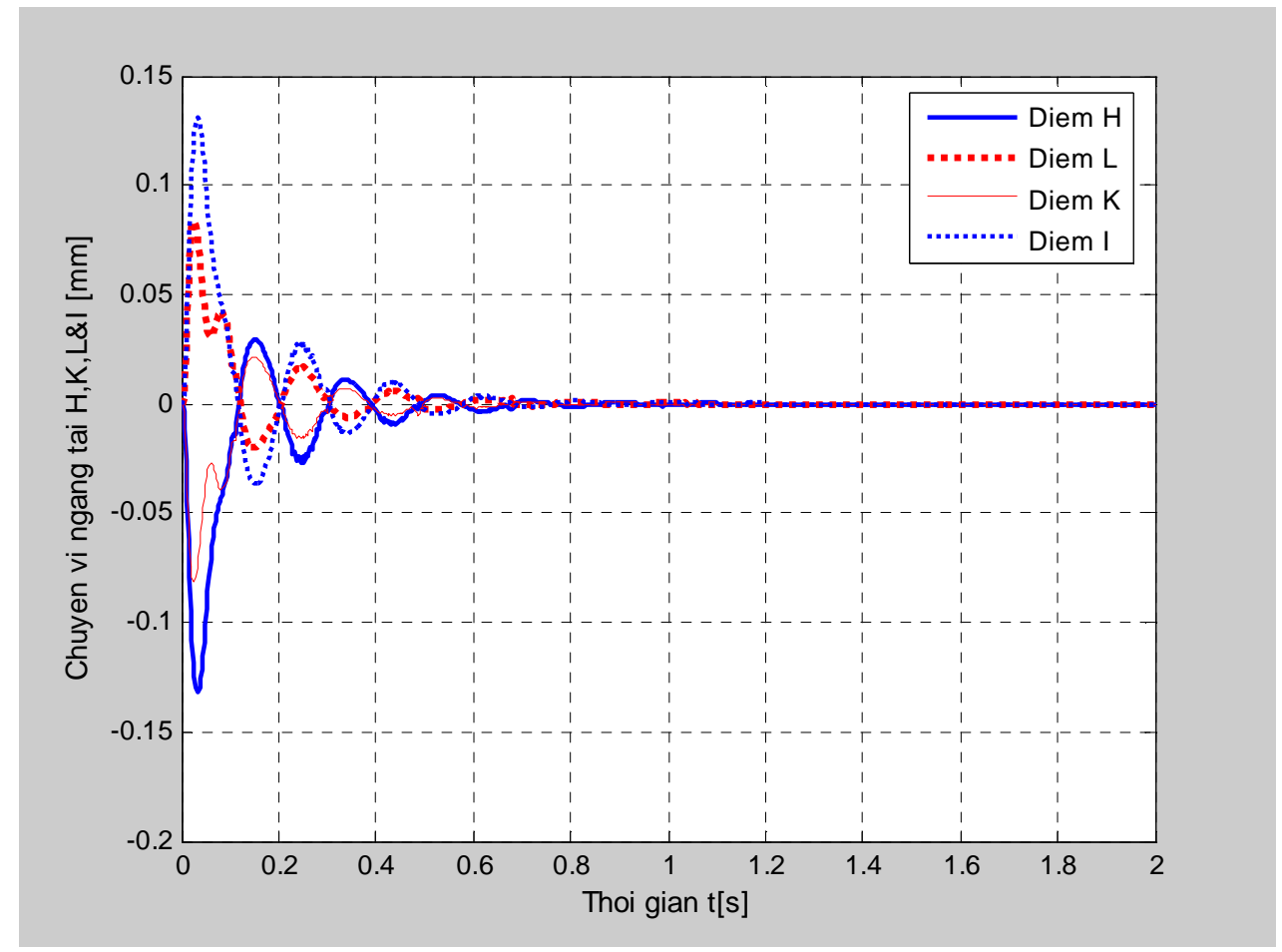

Hình 6: Biến thiên chuyển vị ngang tại điểm $\mathrm{H}, \mathrm{K}, \mathrm{L}$ và I

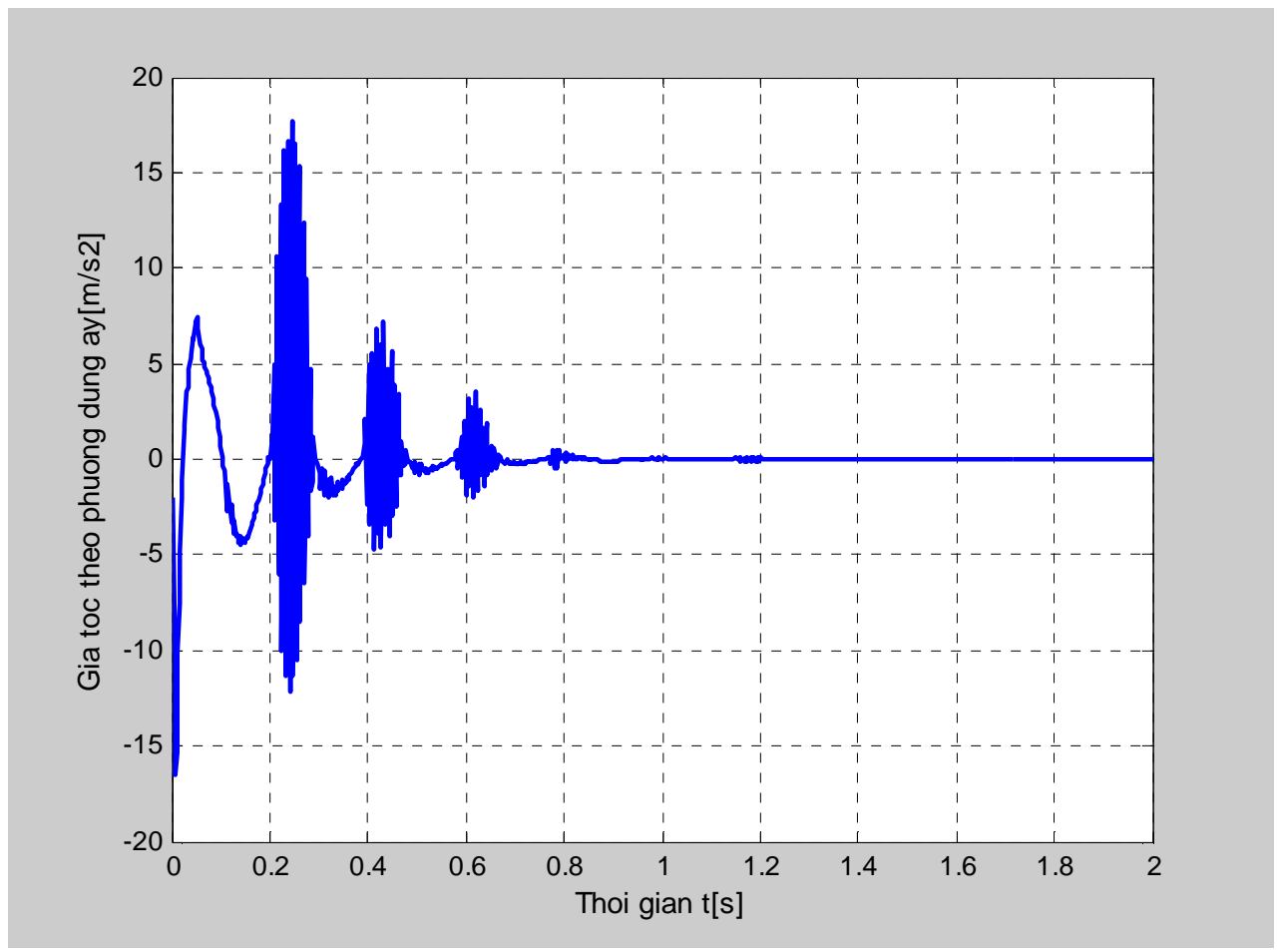

Hình 7: Biến thiên gia tốc tại A 


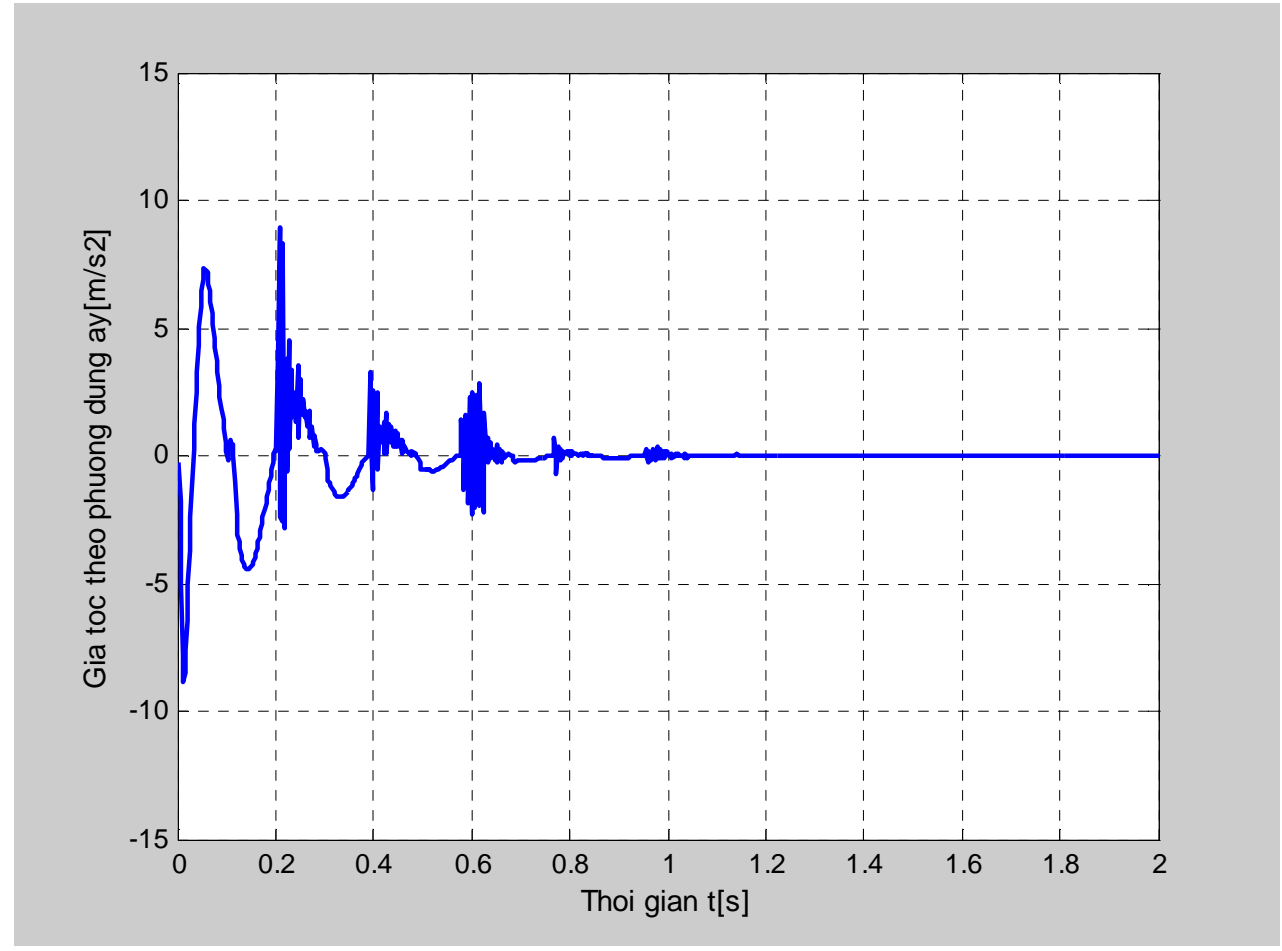

Hình 8: Biến thiên gia tốc tại $\mathrm{C}$

Trên hình 7 và 8 là sự biến thiên gia tốc theo phương đứng của các điểm $\mathrm{A}$ và $\mathrm{C}$ thuộc ống dẫn bên trái.

\section{KẾT LUẬN}

Nội dung bài báo đã đạt được:

- Xây dựng mô hình, thuật toán PTHH và chương trình phần mềm trong môi trường Matlab giải bài toán tương tác giữa hệ ống dẫn và nền san hô chịu tác dụng của tải trọng do nổ của bom đạn gây nên.

- Tính toán trên ví dụ cụ thể, với kết quả chuyển vị, gia tốc tại các điểm tính cho phép lựa chọn các thông số hợp lý thiết kế các công trình dạng này trong nền san hô.

- Kết quả bài báo có thể làm tài liệu tham khảo và là công cụ tính toán tương tác giữa hệ ống dẫn và nền san hô chịu các dạng tải trọng khác nhau.

\section{TÀI LIỆU THAM KHẢO}


1. Hoàng Xuân Lượng, 2010. Nghiên cứu các chỉ tiêu kỹ thuật của nền san hô và tương tác giữa kết cấu công trình và nền san hô, Đề tài cấp Nhà nước, mã số KC.09.07/0610.

2. Bathe K.J and Wilson E.L., 1978. Numerical Method in Finite Method Analyis Prentice, Hall of India Private Limited, New Delhi.

3. Pizhong Qiao, Jialai Wang, 2004. Mechanics and fracture of crack tip deformable bi-material interface, International Journal of Solids and Structures 41 (2004) 7423 7444.

4. F. Zhu \& G. Lu, 2007. A Review of Blast and Impact of Metallic and Sandwich Structures, EJSE Special Issue: Loading on Structures.

\title{
INTERACTION BETWEEN DOUBLE PIPES AND CORAL FOUNDATION UNDER BLAST LOADING
}

\author{
LE TAN
}

Summary: In this study, the author present the calculating method interaction between double pipes and coral foundation under blast loading by finite element method. The problem analysis with using plane strain three nodes triangular element and four nodes $2 D$ slip element (for slip layer between pipes and coral foundation). The paper results can be used in design as reference for the pipes in the coral foundation under blast loading.

Ngày nhận bài: 15 - 10 - 2010

Ngưòi nhận xét: TS. Nguyễn Thái Chung 